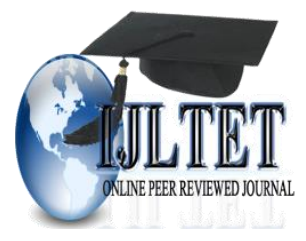

International Journal of Latest Trends in Engineering and Technology

Vol.(9)Issue(2), pp.139-145

DOI: http://dx.doi.org/10.21172/1.92.24

e-ISSN:2278-621X

\title{
FREQUENCY DOMAIN BASED ROBUST DIGITAL WATERMARKING TECHNIQUE FOR VIDEOS
}

\author{
${ }^{1}$ Pradeep Kumar Singh, ${ }^{2}$ Monasa Yengkhom
}

\begin{abstract}
Watermarking is consider as the most promising and emerging technique for the protection of information. Several schemes have been developed and proposed in frequency domain to achieve robustness, imperceptibility, fidelity, etc. Frequency domain has been used as it provides better robustness than other transform methods. So, in this paper, a watermarking scheme for video is proposed using DWT -SVD and by embedding the watermark in areas where scene changes takes place through the use of RGB space along with some logic. Experimental results proved that the proposed scheme is simple but robust against most of the attacks.
\end{abstract}

Keywords - Watermarking, Frequency Domain-Discrete Wavelet Transform (DWT), Singular Value Decomposition (SVD), Scene Change, RGB color space Internet of Things (IoT), Response System, Sensors, Road Accidents

\section{INTRODUCTION}

The development of different type of digital watermarking schemes is mostly due to the advancement in technologies and increase in usage of the internet. Importance are given mostly on achieving the robustness and fidelity of information being transmitted is all due to increase in number of uploads and downloads taking place every day. Some may manipulate the information for their own benefits. Watermarking is a technique that hides an important data within any medium, it may be an image, video or audio. The main goal is to embed the data in such a way it is cannot be easily detected and manipulated by others. It is very similar to techniques of steganography to hide information during different logic. But the differences is that steganography hides information which are similar to the host data whereas watermarking does not require it. Some of the basic information about the steganography is taken from the work of Singh et al.[12]. Some attacks that are considered to check the robustness are rotation, scaling, translation, cropping, image processing attacks, frame dropping, frame swapping, frame averaging, etc.

\section{RELATED WORK}

We have adopted the approached used in the work done by Singh et al. [16] for selecting the most relevant paper for related work. Details mentioned in steganogrpahy based papers are also taken into account [17-18]. Several works have been done in this domain but some of the works that are relevant with the proposed scheme are by J.P.Pandey et al. [1] which uses DWT-SVD as well as RGB-YUV techniques for their watermarking scheme. They have also use a formulae based on 2D systolic arrays for matrix multiplication. Results show that the scheme is effective to robustness and imperceptible. Another work that was referred is based on watermarking in scene based which was introduced by Leelavathy et al. [2]. DMWT (Discrete Multiwavelet Transform) was used along with Quantization index to embed a watermark in uncompressed part of the video. Also, they have used scrambled watermarks along with secret key during the embedding step. Results proved that it is robust against frame swapping, frame averaging and statistical analysis attacks.

${ }^{\mathrm{T}}$ Department of CSE, Jaypee University of Information Technology, Waknaghat Solan (HP), India

${ }^{2}$ Amity School of Engineering \& Technology (ASET), Amity University of Uttar Pradesh, Noida, U.P., INDIA 


\section{FREQUENCY DOMAIN BASED ROBUST DIGITAL WATERMARKING TECHNIQUE FOR VIDEOS}

E.Ganic et al. [3] have also proposed a scheme using DWT-SVD and by allowing modifications in all frequencies. The scheme is robust to almost all kinds of attacks. Another method of using this methods in different way was introduced by Q. Li et al. [4], in this proposed work they have used Human visual model along with the frequency domain technique to increase imperceptibility and robustness in all frequencies and bigger capacity data. Other way to use this technique is by embedding encrypted watermark of singular values into sub-bands of DWT. This method was proposed by M.Zhang et al. [5] and is found to be robust to geometrical attacks. A. Hu et al. [6] also proposed the use of blind watermarking by employing DFT along with DWT in order increase performance in properties like invisibility and robustness. Also, some other watermarking methods were developed to be resistant against degradation attacks [7]. SN. Mali et al. [8] have proposed an algorithm based on scene change by selecting frames for embedding process. It shows good results but shows certain weakness in areas related to motionless. So, S.A.K. Mostafa et al. [9] approach a technique using principal component analysis (PCA) and DWT .It shows robustness and imperceptibility to attacks like histogram equalization, encoding process, etc. Another scheme was proposed in [10] that uses a combination of DCT, DWT and SVD. It employs middle and high frequency bands of DWT and DCT to embed watermark using SVD. Peak signal to noise ratio and mean square error were used to measure the performance. Results proved that the scheme is imperceptible and can maintain the quality of the video. The proposed system is important for the society as it will help in saving the lives of people who die in road accidents just because of the medical help reaching late. The proposed scheme will reduce the number of causalities by providing a system immediate alerts on the smartphone as well as on the cloud when accident happens.

\section{TECHNIQUES}

1. RGB Color Spaces: RGB color space are used by most of the researchers for embedding the watermark [11]. The RGB color space contains three primary colors Red, Green Blue and secondary colors like Cyan, Magenta and Yellow.

2. Discrete Wavelet Transform (DWT): It is the most widely and simplest method in frequency domain which are robust to any attacks [15]. It can decomposed a signal into four components like lower resolution (LL), horizontal (HL), vertical (LH) and diagonal (HH).

3. Singular Value Decomposition (SVD): It is a tool used for converting a set of correlated variables into a set of uncorrelated ones [13]. It is also used for reducing a two dimensional matrix as most of the images are represented in a two dimensional matrix. It is mostly used in image processing because of its properties like transpose and stability etc.

\section{EXPERIMENT \& RESULT}

$A$.

PROPOSED ALGORITHM

- Embedding process

i. Convert the video to frames.

ii. Take R, G, B mean of each frames and analyze the values.

iii. For any drastic change in value, embed a watermark. Embed another watermark for frames which have little changes in their values. Embed another watermark for frames whose values remain the same.

iv. The embedding process uses DWT which divides the plane into four sub-bands.

v. After using DWT, apply SVD over it.

vi. The whole frames are packed into an '.avi' file which results in a watermarked video.

- Watermark Extraction Steps

i. Convert the watermarked video again into frames.

ii. Apply DWT.

iii. Apply SVD.

iv. Extract watermark from each frame and analyze it.

v. The result is the production of 3 different watermarks from each of the frames. 
The Setup method does the following:

- Determines the speed at which the code is supposed to run

- Initializes the accelerometer

- $\quad$ starts the wire port (connected to accelerometer)

B. SIMULATIONS

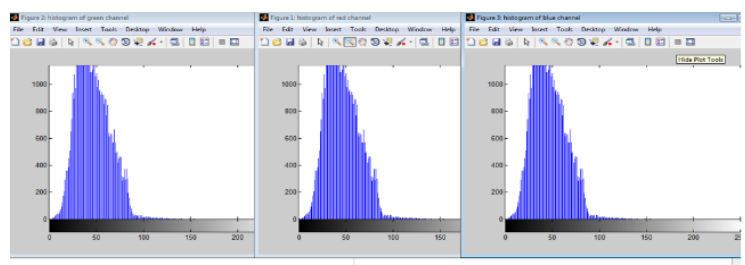

Figure 1

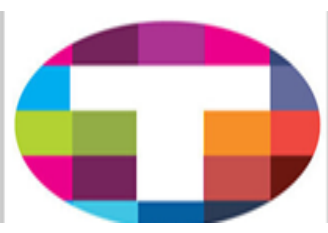

Figure 2 .
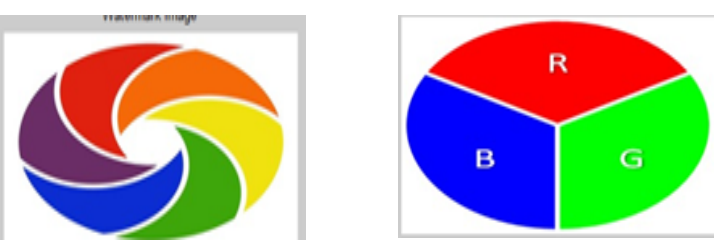

Different watermarks used

Table 1: Mean of some frames

\begin{tabular}{|l|l|l|l|l|} 
Frame name & $\mathrm{R}$ & $\mathrm{G}$ & $\mathrm{B}$ & $\mathrm{R}+\mathrm{G}+\mathrm{B} / 3$ \\
\hline Image 1 & 47.05 & 47.05 & 47.05 & 47.05 \\
\hline Image 3 & 46.67 & 46.67 & 46.67 & 46.67 \\
\hline Image 5 & 46.35 & 46.35 & 46.35 & 46.35 \\
\hline Image 7 & 45.97 & 45.97 & 45.97 & 45.97 \\
\hline Image 9 & 45.71 & 45.71 & 45.71 & 45.71 \\
\hline Image 11 & 45.49 & 45.49 & 45.49 & 45.49 \\
\hline Image 13 & 45.47 & 45.47 & 45.47 & 45.47 \\
\hline Image 15 & 45.49 & 45.49 & 45.49 & 45.49 \\
\hline Image 17 & 45.46 & 45.46 & 45.46 & 45.46 \\
\hline Image 19 & 45.49 & 45.49 & 45.49 & 45.49 \\
\hline
\end{tabular}
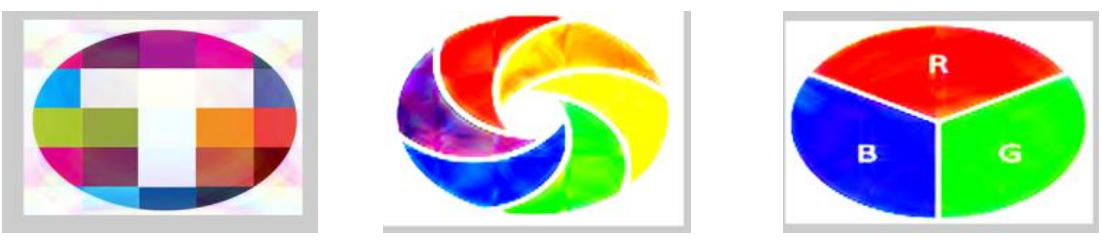

Extracted Watermarks 


\section{RESULTS}

To evaluate the proposed work on basis like robustness and fidelity, some methods were employed which have been discussed below:

i. $\quad$ Fidelity is checked through the use of PSNR.

ii. Robustness is measured by coefficient correlation when the frames undergo attacks like frame averaging, frames tampering, geometric attacks, image processing attacks.

- To check the robustness, the watermarked frames are allowed to undergo some of the attacks like rotation attack, cropping, Gaussian filtering, and frame averaging.
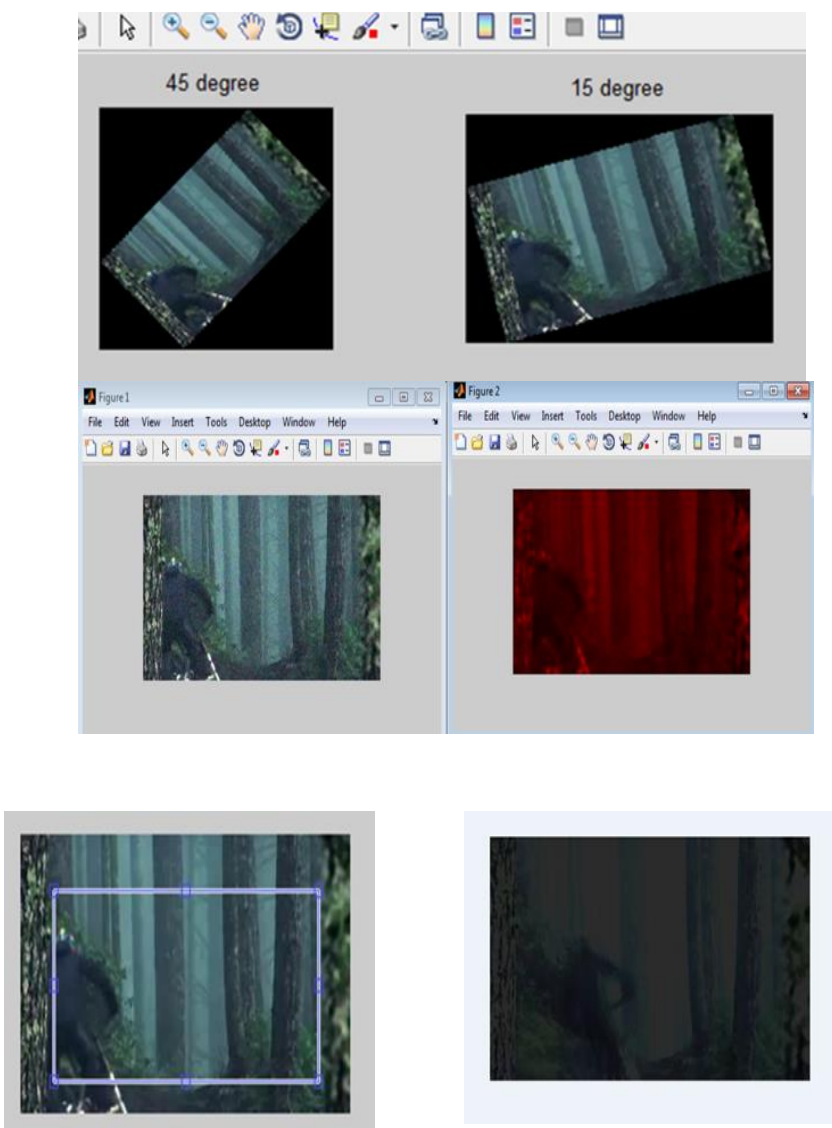

Figure 3. From top to bottom: 45

and 15 degree rotation, Gaussian filtering, cropping, frame Averaging.

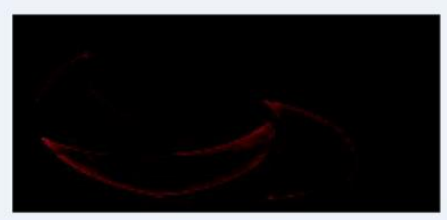

Figure 4. Frame averaging; $7^{\text {th }}$ frame replaced by the average of $7^{\text {th }}, 9$ th and $11^{\text {th }}$ frames 

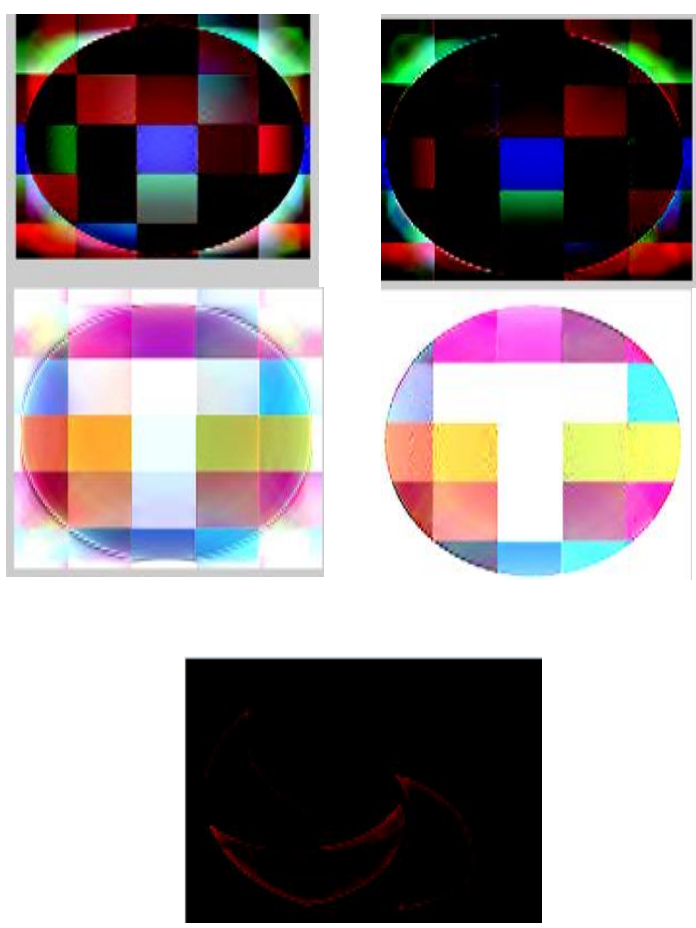

Figure 5. Extracted watermarks from attacked frames.

The Coefficient Correlation (CC) is use to check the robustness. It should lie between -1 to +1 value to prove robustness [14]. The result has been listed below:

Table 2. Values of CC

\begin{tabular}{|c|c|}
\hline Attacks & Coefficient Correlation \\
\hline 45 degree & 0.4 \\
\hline 15 degree & 0.5909 \\
\hline Gaussian filtering & 0.932 \\
\hline cropping & 0.873 \\
\hline Frame averaging & -0.24 \\
\hline
\end{tabular}

Since most of the values lies within the require range, it can be proved that the scheme shows robustness to certain attacks.

- No changes in video could be seen after a frame has been dropped.

- And to check robustness against frame swapping, the 17 th and 18 th frame have been interchanged. No changes were seen.

- Evaluation of PSNR: The fidelity or quality performance of the reconstructed image is usually measured through the use of MSE (Mean Squared error) and PSNR (Peak Signal Noise Ratio). A good quality image should lie in the range of 25 to $50 \mathrm{~dB}$. The results of some frames are shown below: 
Table 3. MSE of some frames

\begin{tabular}{|l|l|l|l|l|}
\hline Frame number & R & G & B & Average \\
\hline 1 & 4.05 & 6.4 & 4.10 & 4.85 \\
\hline 2 & 67.3 & 78.3 & 104.65 & 83.4 \\
\hline 3 & 59.6 & 73.3 & 102.4 & 78.4 \\
\hline 4 & 5.2 & 7.8 & 8.1 & 7.03 \\
\hline 5 & 56.6 & 69.0 & 98.8 & 74.8 \\
\hline 6 & 55.8 & 68.7 & 98.2 & 74.2 \\
\hline
\end{tabular}

Table 4. PSNR of some frames

\begin{tabular}{|l|l|l|l|l|}
\hline $\begin{array}{l}\text { Frame } \\
\text { number }\end{array}$ & R & G & B & Average \\
\hline 1 & 42.08 & 40.08 & 42.03 & 41.40 \\
\hline 2 & 29.88 & 29.22 & 27.96 & 29.02 \\
\hline 3 & 30.40 & 29.50 & 28.50 & 29.46 \\
\hline 4 & 40.9 & 39.2 & 39.05 & 39.71 \\
\hline 5 & 30.67 & 29.79 & 28.24 & 29.56 \\
\hline 6 & 30.67 & 29.79 & 28.24 & 29.56 \\
\hline 7 & 30.80 & 29.86 & 28.27 & 29.64 \\
\hline 8 & 30.80 & 29.86 & 28.27 & 29.64 \\
\hline 9 & 30.86 & 29.80 & 28.23 & 29.63 \\
\hline 10 & 30.91 & 29.77 & 28.18 & 29.62 \\
\hline.. & $\ldots \ldots$ & $\ldots$ & & \\
\hline & & & & $\ldots$ \\
\hline
\end{tabular}

From the average it can be concluded that lower MSE->higher PSNR value and most of the reconstructed image lies within the acceptable range. Thus, most the reconstructed image maintains the quality of the original image.

\section{CONCLUSIONS}

A successful digital watermarking scheme in videos is achieved when it can satisfy all the requirements like robustness, fidelity, imperceptibility, payload etc. For this to achieve, several methods have been experimented on various techniques available based on frequency domain. The proposed scheme is simple, easy and yet fulfill all the qualities of a digital watermarking. It shows robustness and fidelity to almost all the attacks since the results proved that the coefficient correlation and MSE-PSNR lies within the required range. For future prospects, different approaches could be employed to increase the robustness and fidelity in some other attacks including frame averaging.

\section{REFERENCES}

[1] J.P.Pandey , G.Singh, “ Digital Color Image Watermarking using DWT-SVD Techniques in YUV and RGB Color Spaces", International Journal of Advanced Research in Computer Science and Software Engineering, Vol.5, No.1,pp.470-474 2015. 


\section{FREQUENCY DOMAIN BASED ROBUST DIGITAL WATERMARKING TECHNIQUE FOR VIDEOS}

[2] N. Leelavathy, E. V. Prasad and S. Srinivas Kumar, "3D Scene Based Video Watermarking in Discrete Multiwavelet Domain" International Journal of Multidisciplinary Sciences and Engineering, vol.3, No. 7, pp. 12 -17, 2012.

[3] E.Ganic, A.M. Eskicioglu, "Robust DWT-SVD Domain Image Watermarking: Embedding Data in All Frequencies", Proceedings of the 2004 multimedia and security workshop on Multimedia and Security, pp. 166-174, 2004.

[4] Q. Li, C. Yuan; Y. Z. Zhong, “Adaptive DWT-SVD Domain Image Watermarking Using Human Visual Model”, , The 9th International Conference on Advanced Communication Technology ,Vol.3, 1947 - 1951,2007.

[5] M.Zhang, Q. Zhang, C. Zhou "Robust Digital Image Watermarking in DWT-SVD Domain", Artificial Intelligence and Computational Intelligence Lecture Notes in Computer Science, Vol.7003, pp 75-84, 2011.

[6] A.Hu, N. Chen, "A Blind Watermarking Algorithm for Color Image Based on Wavelet Transform and Fourier Transform", published in the proceedings of IEEE 9th International Conference for Young Computer Scientists, ICYCS, Hunan, pp. 1453-1458, 2008.

[7] P. Patil, DS. Bormane, "DWT-based invisible watermarking technique for Digital Images", International journal of Engineering and Advanced Technology, Vol. 2, No. 4, pp.603-605, 2013.

[8] Dr.SN. Mali, C.S. Gosavi, "Frame Selection for Video Watermark Embedding using. Scene Change Detection Algorithm", Technovision-2014, Vol. 5, No. 4, July, 2014.

[9] S.A.K Mostafa, A. S. Tolba, F. M. Abdelkader, H.M. Elhindy, "Video Watermarking Scheme Based on Principal Component Analysis and Wavelet Transform", International Journal of Computer Science and Network Security, IJCSNS, Vol.9 No.8, pp. 45-52,August 2009

[10] C. N. Sujatha, P. Satyanarayana , "High Capacity Video Watermarking based on DWT-DCT-SVD", International Journal of Science, Engineering and Technology Research (IJSETR), Vol. 4, No. 2,pp. 245-249, 2015.

[11] S.Süsstrunk, R.Buckley, S.Swen "Standard RGB color spaces", Proc. IS T/SID 7th Color Imaging Conf., pp.127 -134, 1999.

[12] Pradeep Kumar Singh and Saroha Kriti, A variant of LSB steganography for hiding images in audio, International Journal of Computer Applications, 11.6, 2010.

[13]http://web.mit.edu/be.400/www/SVD/Singular_Value_Decomposition.htm, access on 25/05/15.

[14]http://citeseerx.ist.psu.edu/viewdoc/download?doi=10.1.1.303.6348\&rep=rep1\&type=pdf, access on 26/05/15.

[15] D. Arya, "A survey on frequency and wavelet domain digital watermarking techniques", International Journal of Scientific \& Engineering Research, Vol.1, No. 2, pp. 286-292, 2010.

[16] Singh P. K., Sangwan O. P., \& Sharma A. (2013). A systematic review on fault based mutation testing techniques and tools for Aspect-J programs. In Advance Computing Conference (IACC), 2013 IEEE 3rd International (pp. 1455-1461). IEEE.

[17] Pradeep Kumar Singh, Hitesh Singh, Kriti Saroha, A survey on Steganography in Audio, published in proceeding of the $3^{\text {rd }}$ National Conference-INDIACOM-2009, Computing For Nation Development, BVICAM, Ne w Delhi, 2009. ISSN/ISBN:0973-7529.

[18] Hitesh Singh, Pradeep Kumar Singh, Kriti Saroha, A survey on Text based Steganography, published in proceeding of the $3^{\text {rd }}$ National Conference-INDIACOM-2009, Computing For Nation Development, BVICAM, Ne w Delhi, 2009. ISSN/ISBN:0973-7529. 